\title{
Zinc and ageing (ZINCAGE Project)
}

ZINCAGE is a specific targeted research project (STREP) funded by the European Union in the 6th Framework Program (FP6). It includes epidemiological studies on the influence of diet and lifestyle on healthy ageing, aims at preventing adult degenerative disease and focusses in particular on cardiovascular diseases. Malnutrition in the elderly is also addressed. The ZINCAGE Final Conference was held in Ancona, January 2007 , with papers published in the present Special Issue, and it focussed on the effects of $\mathrm{Zn}$ supplementation in the elderly and on the possible influences of dietary, biochemical and genetic factors on the individual response. $\mathrm{Zn}$ deficiency, cell-mediated immune dysfunction and increased oxidative stress are common in elderly subjects and it is clear that dietary habits including $\mathrm{Zn}$ consumption have a great impact on these factors. Zn supplementation in the elderly can improve the immune response and reduce oxidative stress markers, thereby contributing to a reduced incidence of infections. However, individual differences in the response to $\mathrm{Zn}$ can lead to contradictory results even with supplementation trials performed in elderly people of the same age groups. One of the reasons for such individual differences is the genetic background of the subjects enrolled in the study. In fact, some proteins involved in regulating $\mathrm{Zn}$ homeostasis display polymorphic sites. One important class of such proteins are the metallothioneins (MT), which bind $\mathrm{Zn}$ with high affinity but, at the same time, release free $\mathrm{Zn}$ ions in response to oxidative/nitrosative stress. MT thereby modulate the expression of $\mathrm{Zn}$-dependent genes and activate antioxidant enzymes. Differences in $\mathrm{Zn}$ status have also been observed in individuals carrying different alleles with gene polymorphisms for pro-inflammatory cytokines (i.e. IL-6 and TNF-alpha). In addition, the individual response can be modulated by dietary habits because $\mathrm{Zn}$ absorption and availability is dependent on the intake of other nutrients and trace elements.

So far these aspects have been poorly studied because the intracellular mechanisms involved in the regulation of $\mathrm{Zn}$ homeostasis are not well understood in the context of aging. Moreover, even if it is known that $\mathrm{Zn}$ can modulate antioxidant responses, very little research has been undertaken in order to identify the targets of the intracellular response in ageing. What is known is that during ageing, the intake of $\mathrm{Zn}$ decreases, thus contributing to frailty, general disability and increased incidence of age-related degenerative diseases (cancer, infections and atherosclerosis). To some extent this situation may be of different severity in different European countries, due to the large differences in extrinsic (dietary habits and socio-economic conditions) and intrinsic factors (genetic background) affecting $\mathrm{Zn}$ homeostasis. One of the aims of the ZINCAGE project was to investigate how these factors affect the response to $\mathrm{Zn}$, thus contribute to a better evaluation of the need for this supplement in elderly populations. In the present volume these aspects have been considered in relationship to zinc supplementation in elderly people selected on the basis of the zinc status and specific polymorphisms for IL-6 and/ or MT genes. The results obtained with Zn supplementation in elderly subjects, as presented in this Special Issue, are encouraging. New knowledge on the function of MT and $\mathrm{Zn}$ transporters in ageing has been acquired which can be used to explain the general improvement in immune and antioxidant response observed after zinc supplementation. Many factors were found to affect the individual response to $\mathrm{Zn}$, such as general dietary habits, genotype, gender, drug administration and frailty. This makes it very difficult to draw a simple conclusion regarding the possible benefits of $\mathrm{Zn}$ supplementation during ageing. However, some progress in understanding how $\mathrm{Zn}$ can modulate oxidative stress responses and host defence to infection has been achieved and will hopefully be the subject of further scientific enquiry. It is also clear that in order to clarify many aspects related to the health benefits of $\mathrm{Zn}$, a longitudinal approach will be helpful using cohorts of subjects whose past dietary habits and clinical data can be easily accessed.

All together, this study underlines the importance of nutrition and especially of zinc as determinant of healthy aging. This supports also the need for large collaborative European studies favouring collaboration of various geographical parts and knowledge transfer. 
The Guest Editors Eugenio Mocchegiani

Res. Department INRCA, Immunology Center (Section: Nutrigenomic and Immunosenescence),

Via Birarelli 8, 60121 Ancona, Italy

Tel.: +39 071 8004216; fax: +39071206791.

E-mail address: e.mocchegiani@inrca.it

Alexander Bürkle Chair of Molecular Toxicology, Department of Biology, Box X911, University of Konstanz, D-78457 Konstanz, Germany

Tamas Fulop

Research Center on Aging, Immunology Program,

Geriatric Division, Faculty of Medicine,

University of Sherbrooke, Sherbrooke, Que., Canada 